\title{
Tandem Sonogashira Coupling: an efficient tool for the synthesis of diarylalkynes
}

\author{
Zoltán Novák, Péter Nemes, András Kotschy \\ Department of General and Inorganic Chemistry, \\ Eötvös Loránd University, \\ H-1117 Pázmány Péter sétány 1/A \\ Budapest, Hungary \\ e-mail:kotschy@para.chem.elte.hu
}

\section{Supporting Information}

\section{Contents}

Experimental procedures .....................................................................2

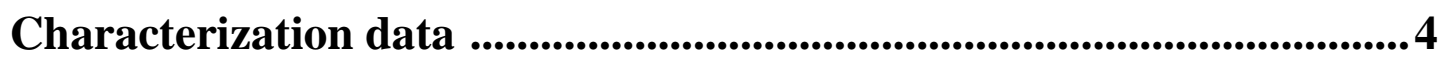

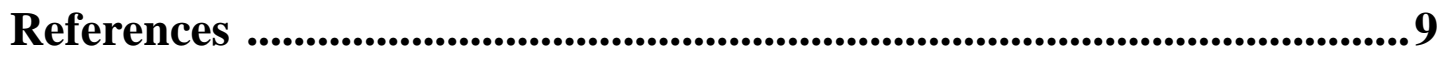

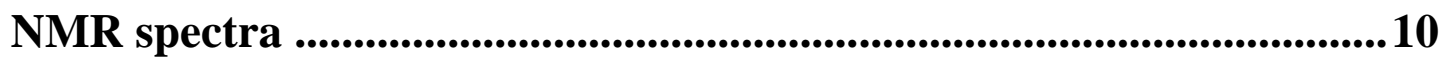




\section{General}

Unless otherwise indicated, all starting materials were obtained from commercial suppliers (Aldrich, Fisher, Merck) and were used without further purification including, dichloromethane, diisopropylamine, n-hexane, toluene, ethyl acetate, $\mathrm{PPh}_{3}, \mathrm{CuI}$, $\mathrm{PdCl}_{2}\left(\mathrm{PPh}_{3}\right)_{2}$, 3-bromopyridine, 3-iodotoluene, iodobenzene, 4-iodoanisole, 2-bromochlorobenzene, 2-methyl-3-butyn-2-ol, $\mathrm{MgSO}_{4}, \mathrm{NaH}$ (55\% in mineral oil), $\mathrm{KOH}$. Toluene and diisopropylamine were purged with argon prior to use. All reactions were performed under an atmosphere of argon. Analytical thin-layer chromatography (TLC) was performed on Polygram SIL G/ UV 254 Pre-coated plastic TLC plates with $0.25 \mathrm{~mm}$ silica gel from Macherey-Nagel + Co. Visualization was performed with a $254 \mathrm{~nm}$ UV lamp. Silica gel column chromatography was carried out with Flash silica gel (0.040 $0.063 \mathrm{~mm}$ ) from Merck. Melting points were determined using a Büchi melting point apparatus. All melting point values are reported as the midpoint of the thermal transition. The ${ }^{1} \mathrm{H}$ and ${ }^{13} \mathrm{C}$ NMR spectra were recorded on a Brucker DRX-250 spectrometer in $\mathrm{CDCl}_{3}$. Chemical shifts are expressed in parts per million $(\delta)$ using residual solvent protons as internal standards: chloroform $\left(\delta 7.26\right.$ for ${ }^{1} \mathrm{H}, \delta 77.00$ for $\left.{ }^{13} \mathrm{C}\right)$. Coupling constants $(J)$ are reported in Hertz $(\mathrm{Hz})$. Splitting patterns are designated as s (singlet), d (doublet), t (triplet), m (multiplet), br s (broad singlet), dd (doublet doublet). Gas chromatographic measurements were performed with a Chrompack CP9001 Gas Chromatograph (10 m x 0.25 mm column with $0.12 \mu \mathrm{m}$ CP-SIL 5CB coating, $\mathrm{H}_{2}$ carrier gas). Combination gas chromatography and low resolution mass spectrometry was obtained on a Hewlett-Packard 5790A Gas Chromatograph (30 m x 0.25 mm column with $0.25 \mu \mathrm{m}$ RH-5 MS+ coating, He carrier gas) and VG 12-250 Mass Spectrometer (Ion source: $\mathrm{EI}+, 70 \mathrm{eV}, 250^{\circ} \mathrm{C}$; interface: $250^{\circ} \mathrm{C}$ ). In the GC measurements di(ethylene glycol) dibutyl ether (40 $\mu$ l per reaction) was used as internal standard.

Method A for the synthesys of diarylakynes: $10 \mathrm{mmol}$ aryl halide, $351 \mathrm{mg}$ $\mathrm{PdCl}_{2}\left(\mathrm{PPh}_{3}\right)_{2}(0,5 \mathrm{mmol}, 5 \%)$ and $95 \mathrm{mg} \mathrm{CuI}$ (0,5 mmol, 5\%) were placed into a flame dried Schlenk flask. $20 \mathrm{~mL}$ diisopropylamine was added to the flask followed by $1260 \mu \mathrm{L}$ 2-methyl-3-butyn-2-ol (13 mmol, 1091mg). The reaction mixture was stirred under argon for one hour at $50^{\circ} \mathrm{C}$. Then 4,48 $\mathrm{g} \mathrm{KOH}(80 \mathrm{mmol}), 351 \mathrm{mg} \mathrm{PdCl}_{2}\left(\mathrm{PPh}_{3}\right)_{2}(0.5 \mathrm{mmol}$, 
5\%), $95 \mathrm{mg}$ CuI (0.5 mmol, 5\%) and $10 \mathrm{mmol}$ aryl halide were added and the rection mixture was heated for five hours in a $110{ }^{\circ} \mathrm{C}$ oil bath. After cooling to room temperature the $\mathrm{KOH}$ was neutralized with $1 \mathrm{M} \mathrm{HCl}$, and then the mixture was extracted with DCM. The combined organic phases were dried over $\mathrm{MgSO}_{4}$ then evaporated. The crude product was purified by column chromatography using hexane-ethyl acetate mixtures.

Method B for the synthesys of diarylakynes: $5 \mathrm{mmol}$ aryl halide, $100 \mathrm{mg} \mathrm{PdCl}_{2}\left(\mathrm{PPh}_{3}\right)_{2}$ (0.14 mmol, 2.8\%) and $28 \mathrm{mg} \mathrm{CuI} \mathrm{(0.14} \mathrm{mmol,} \mathrm{2.8 \% )} \mathrm{were} \mathrm{placed} \mathrm{into} \mathrm{a} \mathrm{flame} \mathrm{dried}$ Schlenk flask. $12 \mathrm{~mL}$ toluene and $1200 \mu \mathrm{L}$ diisopropylamine were added to the flask followed by $630 \mu \mathrm{L}$ 2-methyl-3-butyn-2-ol $(6.5 \mathrm{mmol}, 546 \mathrm{mg})$. The reaction mixture was stirred at $80{ }^{\circ} \mathrm{C}$ under argon until the first coupling was complete. Then the temperature of the oil bath was increased to $110{ }^{\circ} \mathrm{C}$ and $400 \mathrm{mg} \mathrm{NaH}$ (55\% dispersion, $9.16 \mathrm{mmol}$ ) was added slowly to the mixture. After $5 \mathrm{~min}$ stirring $5 \mathrm{mmol}$ of the appropriate aryl halide was added to the reaction mixture, which was followed after 25 min by the careful addition of another $100 \mathrm{mg}$ portion of $\mathrm{NaH}(1.83 \mathrm{mmol})$. The reaction was stirred at $110^{\circ} \mathrm{C}$ until completion (ca. 1hour). After cooling to room temperature the suspension was filtered, and the separated amine-hydrochloride was washed with toluene. Evaporation of the combined toluene solutions gave a crude product, which was purified by column chromatography using hexane-ethyl acetate mixtures.

\section{Combinatorial synthesis by tandem Sonogashira coupling One-pot library synthesis}

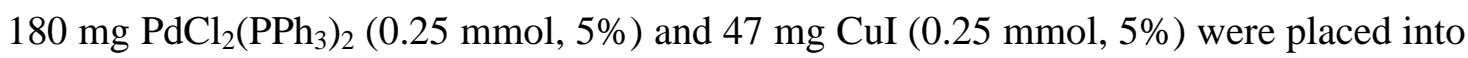
a flame dried Schlenk flask followed by $12 \mathrm{ml}$ diisopropylamine. Then $790 \mathrm{mg}$ (5 mmol) 3-bromopyridine and $532 \mu \mathrm{L}$ 2-methyl-3-butyn-2-ol (5.5 mmol, $462 \mathrm{mg}$ ) was added to the solution. The reaction mixture was stirred at $70{ }^{\circ} \mathrm{C}$ under argon until the first coupling was complete (1 hour). Then $180 \mathrm{mg} \mathrm{PdCl}\left(\mathrm{PPh}_{3}\right)_{2}$ (0.25 mmol, 5\%), $47 \mathrm{mg} \mathrm{CuI}$ (0.25 mmol, 5\%), $186 \mu \mathrm{L}$ (1.66 mmol, $339 \mathrm{mg}$ ) iodobenzene, $213 \mu \mathrm{L}$ (1.66 mmol, $362 \mathrm{mg}$ ) 3iodotoluene, and $388 \mathrm{mg}$ (1.66 mmol) 4-iodoanosole was added to the mixture followed by $2.24 \mathrm{~g} \mathrm{KOH}$. The Schlenk flask was purged again with argon, sealed and heated in ane oil bath at $110{ }^{\circ} \mathrm{C}$ until completion. 


\section{Two-step library synthesis}

$90 \mathrm{mg} \mathrm{PdCl} 2\left(\mathrm{PPh}_{3}\right)_{2}(0.0125 \mathrm{mmol}, 5 \%)$ and $24 \mathrm{mg} \mathrm{CuI} \mathrm{(0.0125} \mathrm{mmol,} \mathrm{5 \% )} \mathrm{were} \mathrm{placed}$ into a flame dried Schlenk flask followed by $6 \mathrm{~mL}$ diisopropylamine, $402 \mathrm{mg}$ ( $2.5 \mathrm{mmol}$ ) 4-(3'-pyridyl)-2-methyl-3-butyn-2-ol, $93 \mu \mathrm{L}$ (0.83 mmol, $169.5 \mathrm{mg})$ iodobenzene, 107 $\mu \mathrm{L}$ (0.83 mmol, $181 \mathrm{mg}$ ) 3-iodotoluene, $194 \mathrm{mg}$ (0.83 mmol) 4-iodoanosole and $1.12 \mathrm{~g}$ $\mathrm{KOH}$ (8eq.) under argon. Then the Schlenk flask was placed into the oil bath which was preheated to $110{ }^{\circ} \mathrm{C}$ and the reaction was stirred until completion. Samples were taken off, diluted with ether and examined with GC.

\section{Characterization of the products}

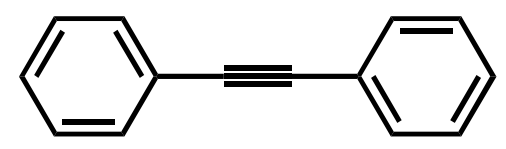

Diphenylacetylene (4a) ${ }^{\mathbf{1}}$ : Yield: Method A: 1317mg (7.4 mmol, 74\% ); Method B: 498 mg (2.8 mmol, 56\% ) white crystal. m.p.: $60-61{ }^{\circ} \mathrm{C} .{ }^{1} \mathrm{H}$ NMR $\left(\mathrm{CDCl}_{3} ; 250 \mathrm{MHz}\right): \delta 7.59$ 7.55 (m, 4H), 7.39-7.36 (m, 6H); ${ }^{13} \mathrm{C} \mathrm{NMR}\left(\mathrm{CDCl}_{3}, 62.5 \mathrm{MHz}\right): \delta 131.6,128.3,128.2$, 123.2, 89.4; GC-MS (EI, 70eV) m/z C ${ }_{14} \mathrm{H}_{10}$ (\% relative intensity, ion): 178 (100, [M] ${ }^{+}$), 176 (61), 152 (40), 126 (29), 89 (23), 76 (68).

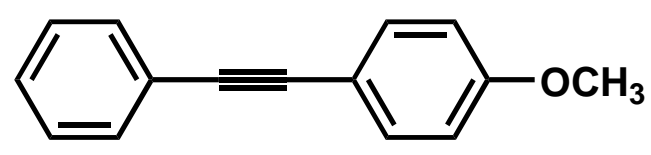

1-Methoxy-4-(phenylethynyl)-benzene (4b) ${ }^{2}$ : Yield: Method A: $603 \mathrm{mg}$ (2.9 mmol, 29\% ), 1331 mg (6.4 mmol, 64\%), Method B: 582 mg (2.8 mmol, 56\% ), 707 mg (3.4 mmol, 68\%) white crystal. m.p.: 79-81 ${ }^{\circ} \mathrm{C} .{ }^{1} \mathrm{H}$ NMR $\left(\mathrm{CDCl}_{3} ; 250 \mathrm{MHz}\right): \delta$ 7.56-7.48 (m, 4H), 7.36-7.33 (m, 3H), 6.80 (dd, $1 \mathrm{H}, J=4.2,2.0 \mathrm{~Hz}), 3.83$ (s, 3H). ${ }^{13} \mathrm{C} \mathrm{NMR}\left(\mathrm{CDCl}_{3}\right.$, $62.5 \mathrm{MHz}): \delta$ 159.2, 133.0, 131.4, 128.3, 127.9, 123.5, 115.2, 113.9, 89.3, 88.0, 55.2 MS(EI, 70eV) m/z C C ${ }_{15} \mathrm{H}_{12} \mathrm{O}$ (\% relative intensity, ion): 208 (100, [M] $\left.]^{+}\right), 193$ (82), 165 (68), 139 (25). 


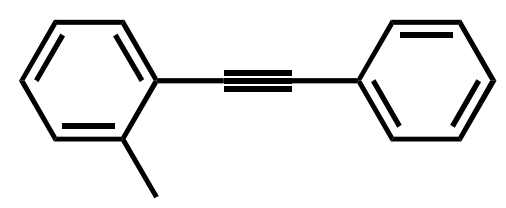

1-Methyl-3-(phenylethynyl)-benzene (4c) ${ }^{3}$ : Yield: Method B: 662 mg (3.45 mmol, 69\%

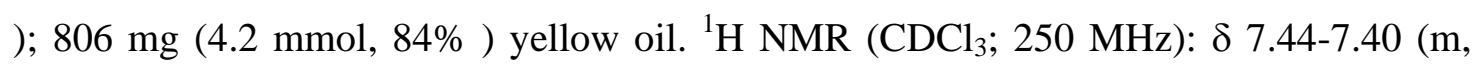
2H), 7.26-7.20 (m, 5H), 7.11 (t, $1 \mathrm{H}, J=8.0 \mathrm{~Hz}$ ), 7.03 (d, 1H, $J=8.0 \mathrm{~Hz}$ ), 2.23 (s, 3H). ${ }^{13} \mathrm{C} \mathrm{NMR}\left(\mathrm{CDCl}_{3}, 62.5 \mathrm{MHz}\right): \delta 137.8,131.6,129.1,128.7,128.6,128.4,128.3,128.2$, 123.4, 123.0, 89.6, 89.0, 21.2. MS(EI, 70eV) m/z C ${ }_{15} \mathrm{H}_{12}$ (\% relative intensity, ion): 192 (100, [M] $]^{+}, 165$ (18), 139 (7), 139 (8), 115 (10).

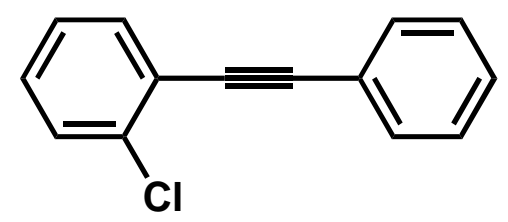

1-Chloro-2-(phenylethynyl)-benzene (4d) ${ }^{2}$ : Yield: Method A: 806 mg (3.8 mmol, 38\% ); Method B: 508 mg (2.4 mmol, 47 \% ), 763 mg (3.6 mmol, $71 \%$ ) yellow oil. ${ }^{1} \mathrm{H}$ NMR $\left(\mathrm{CDCl}_{3} ; 250 \mathrm{MHz}\right): \delta$ 7.50-7.43 (m, 3H), 7.29-7.22 (m, 3H), 7.14-7.10 (m, 3H). ${ }^{13} \mathrm{C}$ NMR ( $\left.\mathrm{CDCl}_{3}, 62.5 \mathrm{MHz}\right): \delta 135.8,133.2,131.7,131.5,129.22,129.20,128.6,128.32$, 128.29, 126.4, 123.1, 122.8, 94.5, 86.1 MS(EI, 70eV) m/z C ${ }_{14} \mathrm{H}_{9} \mathrm{Cl}$ (\% relative intensity, ion): 212(100, $\left.[\mathrm{M}]^{+}\right), 153(11), 126(21)$.

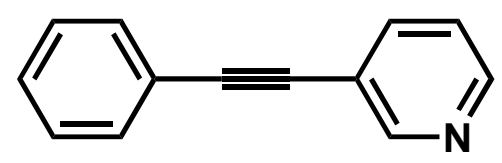

3-Phenylethynyl-pyridyn (4e) ${ }^{4}$ : Yield: Method A: $1002 \mathrm{mg}$ (5.6 mmol, 56\% ) yellow crystal. m.p.: 50-51 ${ }^{\circ} \mathrm{C} .{ }^{1} \mathrm{H}$ NMR $\left(\mathrm{CDCl}_{3}\right.$; $\left.250 \mathrm{MHz}\right): \delta 8.7$ (d, $\left.1 \mathrm{H}, J=1.5 \mathrm{~Hz}\right), 8.49$ (dd, $1 \mathrm{H}, J=5.0,1.5 \mathrm{~Hz}$ ), 7.77 (dt, $1 \mathrm{H}, J=1.75,8.0 \mathrm{~Hz}$ ), 7.50-7.46 (m, 2H), 7.33-7.28 (m, 3H), 7.25-7.19 (m, 1H). ${ }^{13} \mathrm{C} \mathrm{NMR}\left(\mathrm{CDCl}_{3}, 62.5 \mathrm{MHz}\right): \delta 151.9,148.3,138.6,131.7$, 
128.8, 128.4, 123.1, 122.4, 92.8, 85.8 MS(EI, 70eV) m/z C ${ }_{13} \mathrm{H}_{9} \mathrm{~N}$ (\% relative intensity, ion): 179(100, [M] $\left.]^{+}\right), 153(11), 126(21)$.

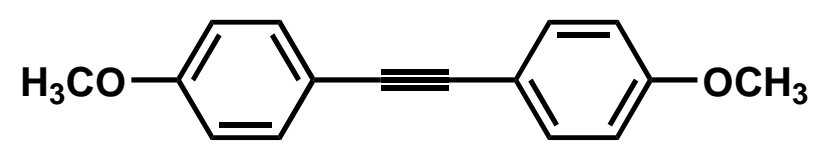

1-Methoxy-4-(4'-methoxy- phenylethynyl)-benzene (4f) ${ }^{\mathbf{1}}$ : Yield: Method A: $603.2 \mathrm{mg}$ (2.9 mmol, 29\% ); Method B: 785 mg (3.3 mmol, 66 \% ), yellow crystal. m.p.: 140-142 ${ }^{\circ} \mathrm{C} .{ }^{1} \mathrm{H} \mathrm{NMR}\left(\mathrm{CDCl}_{3} ; 250 \mathrm{MHz}\right): \delta$ 7.39-7.36 (m, 4H), 6.89-6.69 (m, 4H) 3.74 (s, 6H). ${ }^{13} \mathrm{C} \mathrm{NMR}\left(\mathrm{CDCl}_{3}, 62.5 \mathrm{MHz}\right): \delta 159.3,133.7,114.8,113.1,88.0,56.4 \mathrm{MS}(\mathrm{EI}, 70 \mathrm{eV}) \mathrm{m} / \mathrm{z}$ $\mathrm{C}_{16} \mathrm{H}_{14} \mathrm{O}_{2}$ (\% relative intensity, ion): 238(100), [M] $]^{+}$), 223(84), 180(16), 151(17), 119(20).

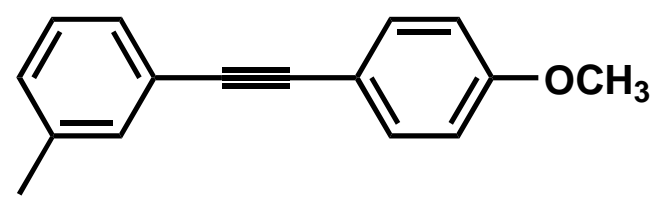

1-Methyl-3-(4'-methoxyphenyl)-benzene (4g): Yield: Method B: 877 mg (3.95 mmol, $79 \%$ ), 733 mg (3.3 mmol, 66\% ) yellow crystal. m.p.:43-45 ${ }^{\circ} \mathrm{C} .{ }^{1} \mathrm{H}$ NMR $\left(\mathrm{CDCl}_{3} ; 250\right.$ MHz): $\delta 7.47$ (d, 2H, $J=8.75 \mathrm{~Hz}$ ), 7.33 (d, 2H, $J=8.5 \mathrm{~Hz}), 7.21$ (t, 1H, $J=6.75 \mathrm{~Hz}$ ), 7.13 (d, 1H, $J=7.5 \mathrm{~Hz}$ ), 6.88 (d, 2H, $J=8.75 \mathrm{~Hz}$ ), 3.80 (s, 3H), 2.34 (s, 3H). ${ }^{13} \mathrm{C}$ NMR $\left(\mathrm{CDCl}_{3}, 62.5 \mathrm{MHz}\right): \delta 159.5,137.9,133.0,132.0,128.8,128.5,128.2,123.4,115.5$, 114.0, 89.0, 88.2, 55.2, 21.2. $\mathrm{MS}(\mathrm{EI}, 70 \mathrm{eV}) \mathrm{m} / \mathrm{z} \mathrm{C}_{16} \mathrm{H}_{14} \mathrm{O}$ (\% relative intensity, ion): 222(100, [M] $\left.]^{+}\right), 207(48), 178(31), 151(9)$.

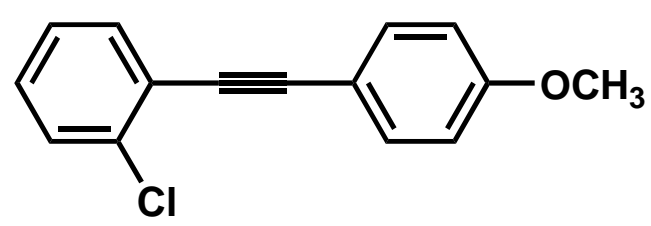


1-Methoxy-4-(2'-chloro- phenylethynyl)-benzene (4h): Yield: Method A: $1645 \mathrm{mg}$ (6.8 mmol, 68\% ); Method B: 509 mg (2.1 mmol, 41 \% ), $921 \mathrm{mg}$ (3.8 mmol, 75 \%) yellow crystal. m.p.: $52-54{ }^{\circ} \mathrm{C} .{ }^{1} \mathrm{H}$ NMR $\left(\mathrm{CDCl}_{3} ; 250 \mathrm{MHz}\right): \delta 7.45-7.41$ (m, 3H), 7.35 (d, $1 \mathrm{H}, \mathrm{J}=9.5 \mathrm{~Hz}), 7.16$ (d, $2 \mathrm{H}, J=9.5 \mathrm{~Hz}), 6.82$ (d, $2 \mathrm{H}, J=8.75 \mathrm{~Hz}) 3.74$ (s, $3 \mathrm{H}) .{ }^{13} \mathrm{C}$ NMR $\left(\mathrm{CDCl}_{3}, 62.5 \mathrm{MHz}\right): \delta$ 159.9, 133.2, 133.0, 129.2, 128.9, 127.1, 126.4, 123.5, 114.9, 114.0, 94.6, 84.9, 55.3. MS(EI, 70eV) m/z C ${ }_{15} \mathrm{H}_{11} \mathrm{OCl}$ (\% relative intensity, ion): 244 ((31), [M] $\left.]^{+}\right), 242$ ((100), [M] $\left.]^{+}\right), 226(59), 198$ (47). Analysis $\left(\mathrm{C}_{15} \mathrm{H}_{11} \mathrm{ClO}\right)$ calculated: C, 74.23; H, 4.57; Found: C, 74.11; H, 4.40.

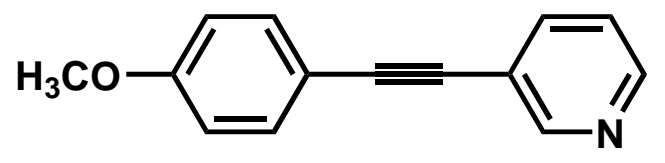

1-Methoxy-4-(3'-pyridynethynyl)-benzene (4i): Yield: Method A: 1359 mg (6.5 mmol, $65 \%$ ) yellow crystal. m.p.: 46-48 ${ }^{\circ} \mathrm{C} .{ }^{1} \mathrm{H}$ NMR $\left(\mathrm{CDCl}_{3} ; 250 \mathrm{MHz}\right): \delta 8.67$ (s, $\left.1 \mathrm{H}\right), 8.44$ (d, 1H, $J=3.75 \mathrm{~Hz}$ ), 7.71 (dt, 1H, $J=8.0,2.0 \mathrm{~Hz}$ ) 7.43-7.37 (m, 2H), 7.20-7.17 (m, 1H), 6.83-6.79 (m, 2H). ${ }^{13} \mathrm{C} \mathrm{NMR}\left(\mathrm{CDCl}_{3}, 62.5 \mathrm{MHz}\right): \delta$ 159.9, 151.9, 148.1, 138.2, 133.1, 123.0, 114.4, 114.0, 92.7, 84.6, 55.2. MS(EI, 70eV) m/z C ${ }_{14} \mathrm{H}_{11} \mathrm{NO}$ (\% relative intensity, ion): 209 (100), [M] $]^{+}$), 194 (91), 166 (46), 139 (60), 113 (25). Analysis $\left(\mathrm{C}_{14} \mathrm{H}_{11} \mathrm{NO}\right.$ ) calculated: C, 80.36; H, 5.30; N, 6.69. Found: C, 80.45; H, 5.29; N, 6.84.

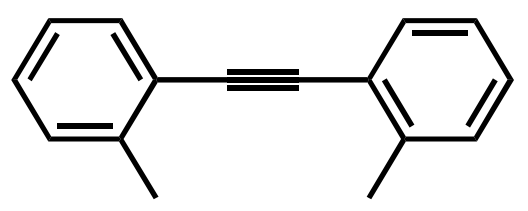

1-Methyl-3-(3’-methylphenyl)-benzene (4j) ${ }^{\mathbf{5}}$ : Yield: Method B: $803 \mathrm{mg}$ (3.9 mmol, $78 \%$ ) white crystal. m.p.: 57-59 ${ }^{\circ} \mathrm{C} .{ }^{1} \mathrm{H}$ NMR $\left(\mathrm{CDCl}_{3} ; 250 \mathrm{MHz}\right): \delta 7.34$ (d, 4H, $J=7.75$ $\mathrm{Hz}), 7.20$ (t, 2H, $J=7.5 \mathrm{~Hz}$ ), 7.12 (d, $2 \mathrm{H}, J=7.5 \mathrm{~Hz}), 2.32$ (s, 6H). ${ }^{13} \mathrm{C} \mathrm{NMR}\left(\mathrm{CDCl}_{3}\right.$, 62.5 MHz): $\delta$ 137.9, 132.1, 129.0, 128.6, 128.2, 123.1, 89.2, $21.2 \mathrm{MS}(\mathrm{EI}, 70 \mathrm{eV}) \mathrm{m} / \mathrm{z}$ $\mathrm{C}_{16} \mathrm{H}_{14}$ (\% relative intensity, ion): 206 (100, [M] ${ }^{+}$), 189 (25), 178 (10), 165 (9), 139 (8). 


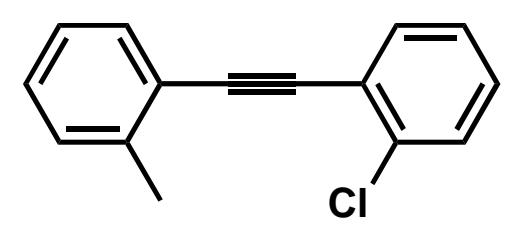

1-Methyl-3-(2'-chloro- phenylethynyl)-benzene (4k): Yield: Method B: 727 mg (2.95 mmol, 59\% ), $493 \mathrm{mg}$ (2.0 mmol, 40\% ) yellow crystal. m.p.: $39-41^{\circ} \mathrm{C} .{ }^{1} \mathrm{H}$ NMR $\left(\mathrm{CDCl}_{3}\right.$; $250 \mathrm{MHz}): \delta 7.53$ (t, 1H, $J=5.0 \mathrm{~Hz}$ ), 7.42-7.36 (m, 3H), 7.26-7.12 (m, 4H), 2.33 (s, 3H). ${ }^{13} \mathrm{C} \mathrm{NMR}\left(\mathrm{CDCl}_{3}, 62.5 \mathrm{MHz}\right): \delta$ 138.0, 135.8, 133.1, 132.2, 129.5, 129.2, 128.8, 126.3, 123.2, 122.6, 94.7, 85.8, 21.2. $\mathrm{MS}(\mathrm{EI}, 70 \mathrm{eV}) \mathrm{m} / \mathrm{z} \mathrm{C}_{15} \mathrm{H}_{11} \mathrm{Cl}$ (\% relative intensity, ion): 228 (25, [M] $\left.]^{+}\right), 226$ (100, [M] $\left.]^{+}\right), 189$ (50), 165 (11), 113 (10), 94 (18).

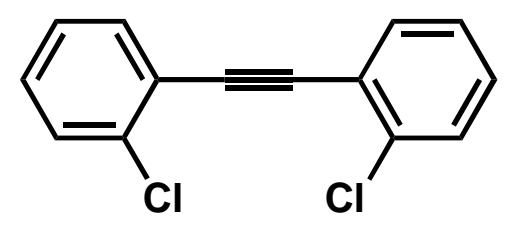

1-Chloro-2-(2'-chlorophenyl-ethynyl)-benzene (4l) ${ }^{1}$ : Yield: Method A: $1641 \mathrm{mg}$ (6.7 mmol, 67\% ); Method B: 395 mg (1.6 mmol, 32 \% ) yellow crystal. m.p.: 83-85 ${ }^{\circ} \mathrm{C} .{ }^{1} \mathrm{H}$ NMR ( $\mathrm{CDCl}_{3}$; $\left.250 \mathrm{MHz}\right): \delta$ 7.54-7.51 (m, 2H), 7.38-7.34 (m, 2H), 7.21-7.16 (m, 4H). ${ }^{13} \mathrm{C}$ NMR $\left(\mathrm{CDCl}_{3}, 62.5 \mathrm{MHz}\right): \delta$ 136.0, 133.4, 129.6, 129.3, 126.4, 122.8, 91.1. MS(EI, $70 \mathrm{eV}) \mathrm{m} / \mathrm{z} \mathrm{C}_{14} \mathrm{H}_{8} \mathrm{Cl}_{2}$ (\% relative intensity, ion): 248 (30, $\left.[\mathrm{M}]^{+}\right), 245$ (100, [M] $\left.]^{+}\right), 211$ (7), 209 (13), 176 (15), 175 (17), 174 (10), 150 (119), 123 (16).

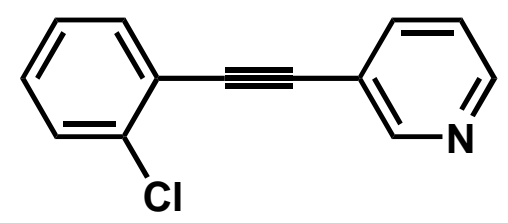

2-(3’-Pyridylethynyl)-chlorobenzene (4m): Yield: Method A: $1320 \mathrm{mg}$ (6.2 mmol, 62\% ) yellow oil. ${ }^{1} \mathrm{H} \mathrm{NMR}\left(\mathrm{CDCl}_{3} ; 250 \mathrm{MHz}\right): \delta 8.7$ (d, $\left.1 \mathrm{H}, J=1.75 \mathrm{~Hz}\right), 8.46$ (dd, $1 \mathrm{H}, J=$ 5.0, $1.75 \mathrm{~Hz}$ ), 7.74 (dt, $1 \mathrm{H}, J=7.25,2.5 \mathrm{~Hz}$ ) 7.47-7.43 (m, 1H), 7.34-7.30 (m, 1H), 7.207.10 (m, 3H). ${ }^{13} \mathrm{C} \mathrm{NMR}\left(\mathrm{CDCl}_{3}, 62.5 \mathrm{MHz}\right): \delta$ 152.0, 148.7, 138.4, 135.8, 133.1, 129.7, 
129.2, 126.4, 122.9, 122.3, 119.9, 90.8, 89.2. MS(EI, 70eV) m/z C ${ }_{13} \mathrm{H}_{8} \mathrm{ClN}$ (\% relative intensity, ion): 215 ((32), [M] $\left.]^{+}\right), 213$ ((100), [M] $\left.]^{+}\right), 178$ (23), 151 (29), 150 (17), 125 (20). Analysis $\left(\mathrm{C}_{13} \mathrm{H}_{8} \mathrm{ClN}\right)$ calculated: $\mathrm{C}, 73.08$; H, 3.77; N, 6.56; Found: C, 73.21; H, 3.73; N, 6.32;

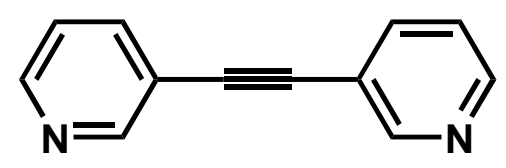

3-(3’-Pyridylethynyl)-pyridin (4n) ${ }^{6}$ : Yield: Method A: 1512 mg (8.4 mmol, 84\% ) yellow crystal. m.p.: ${ }^{\circ} \mathrm{C} .{ }^{1} \mathrm{H}$ NMR $\left(\mathrm{CDCl}_{3} ; 250 \mathrm{MHz}\right): \delta 8.72(\mathrm{~d}, 2 \mathrm{H}, J=1.75 \mathrm{~Hz}), 8.53$ (dd, 2H, $J=5.0,1.75 \mathrm{~Hz}), 7.79(\mathrm{dt}, 2 \mathrm{H}, J=7.25,2.5 \mathrm{~Hz}) 7.28-7.22(\mathrm{~m}, 2 \mathrm{H}) .{ }^{13} \mathrm{C} \mathrm{NMR}$ $\left(\mathrm{CDCl}_{3}, 62.5 \mathrm{MHz}\right): \delta$ 152.1, 148.9, 138.6, 123.1, 119.7, 89.1. MS(EI, 70eV) m/z $\mathrm{C}_{12} \mathrm{H}_{8} \mathrm{~N}_{2}$ (\% relative intensity, ion): 180 ((100), [M] $]^{+}$), 152 (15), 127 (11), 100 (18).

\section{References}

1 Mio, M. J.; Kopel, L. C.; Braun, J. B.; Gidzikwa, T. L.; Hull, K. L.; Brisbois, R. G.; Markworth, C. J.; Grieco, P. A.; Org. Lett. 2002, 4, 3199.

2 Mouriés, V.; Waschbüsch, R.; Carran, J.; Savignac, P.; Synthesis 1998, 271.

3 Eberhard, M. R.; Wang, Z.; Jensen, C. M.; Chem. Comm. 2002, (8), 818.

4 Mori, Y.; Seki, M.; J. Org. Chem. 2003, 68, 1517.

5 Newkome, G. R.; Roper, J. M.; Robinson, J. M.; J. Org. Chem. 1980, 45, 4380.

6 Negishi, E.-I.; Caiding, X.; Ze, T.; Martin, K. Heterocycles 1997, 46, 209. 

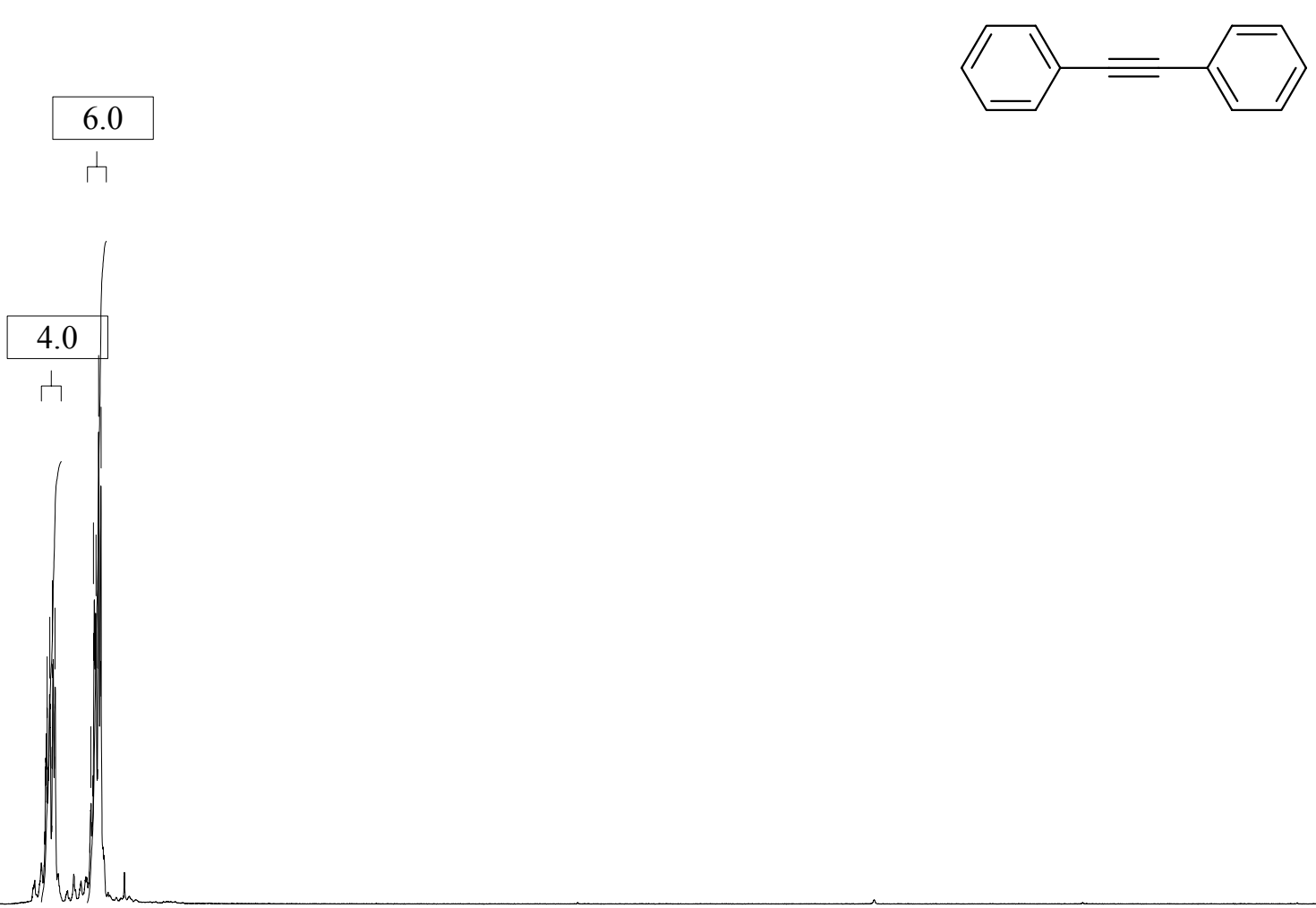


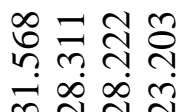

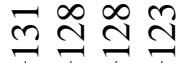

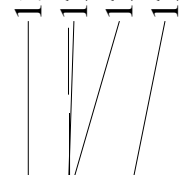

m.

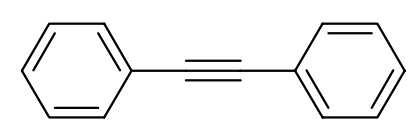




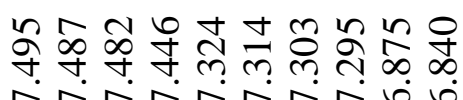

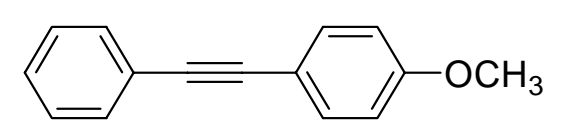




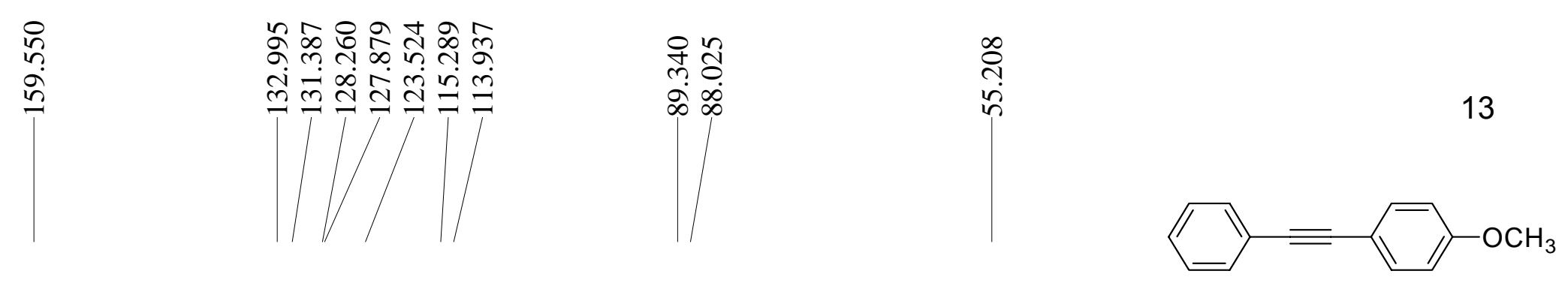




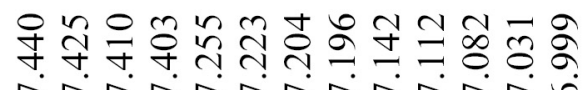

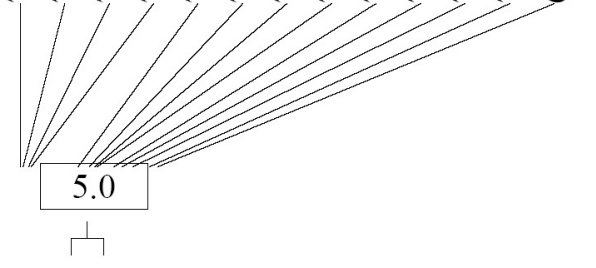

$$
\beta=0
$$
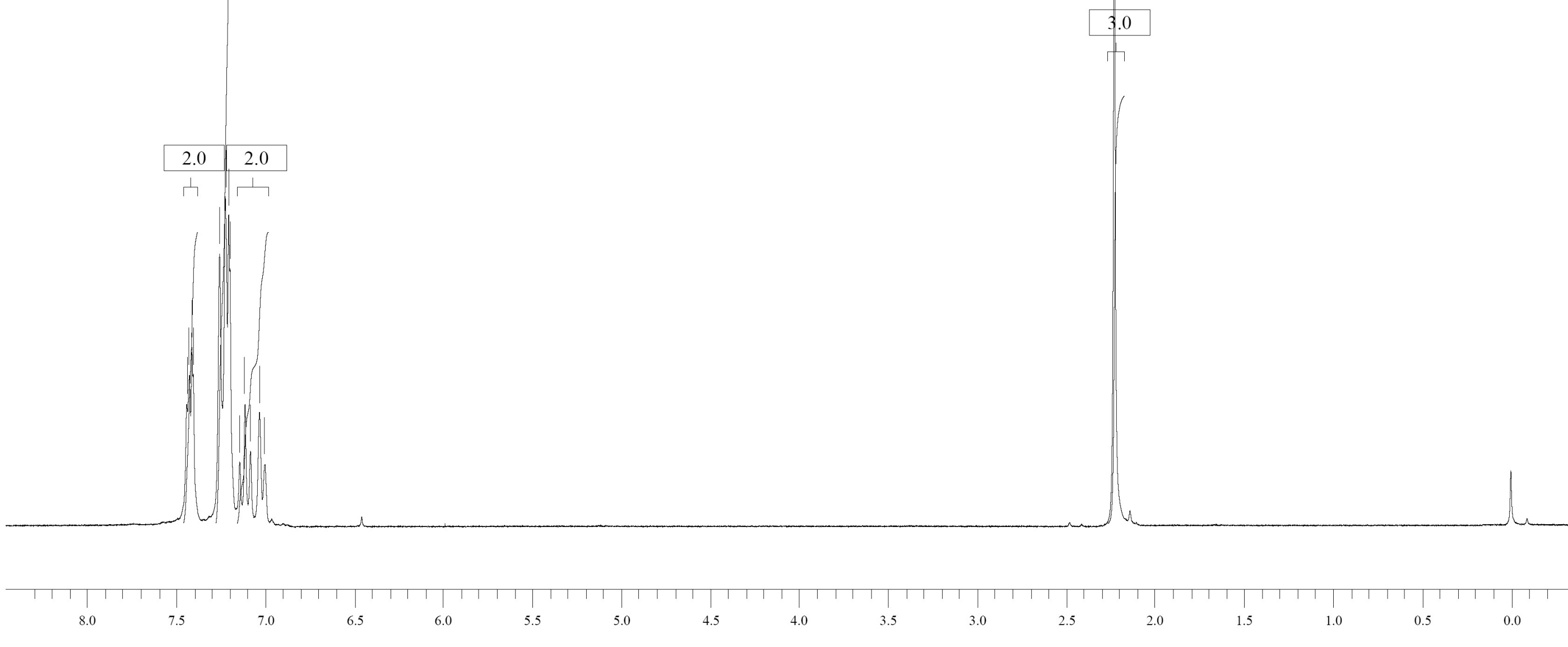
స్త

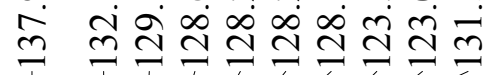

ถ⿻ำ

$\stackrel{+}{\rightarrow}$

के $\infty$

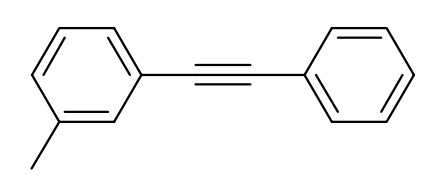




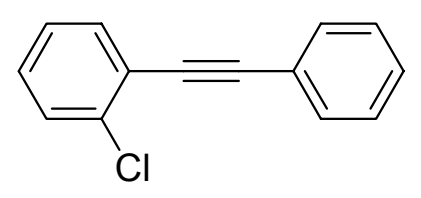




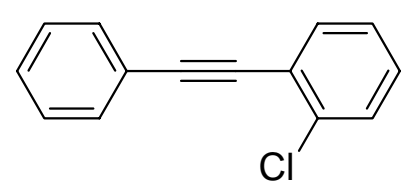




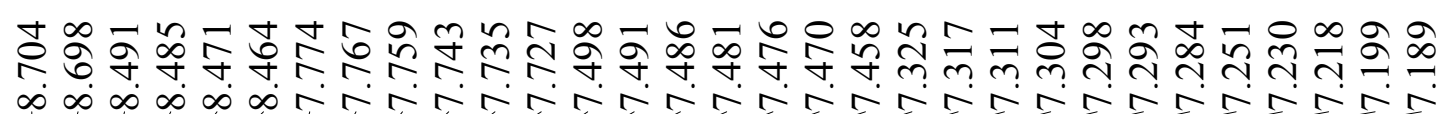

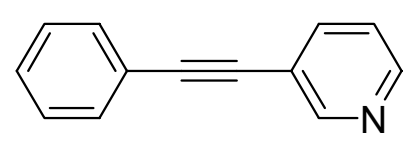




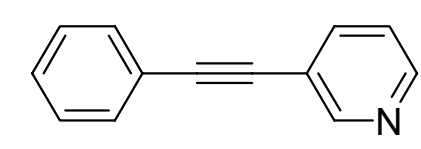

mom 


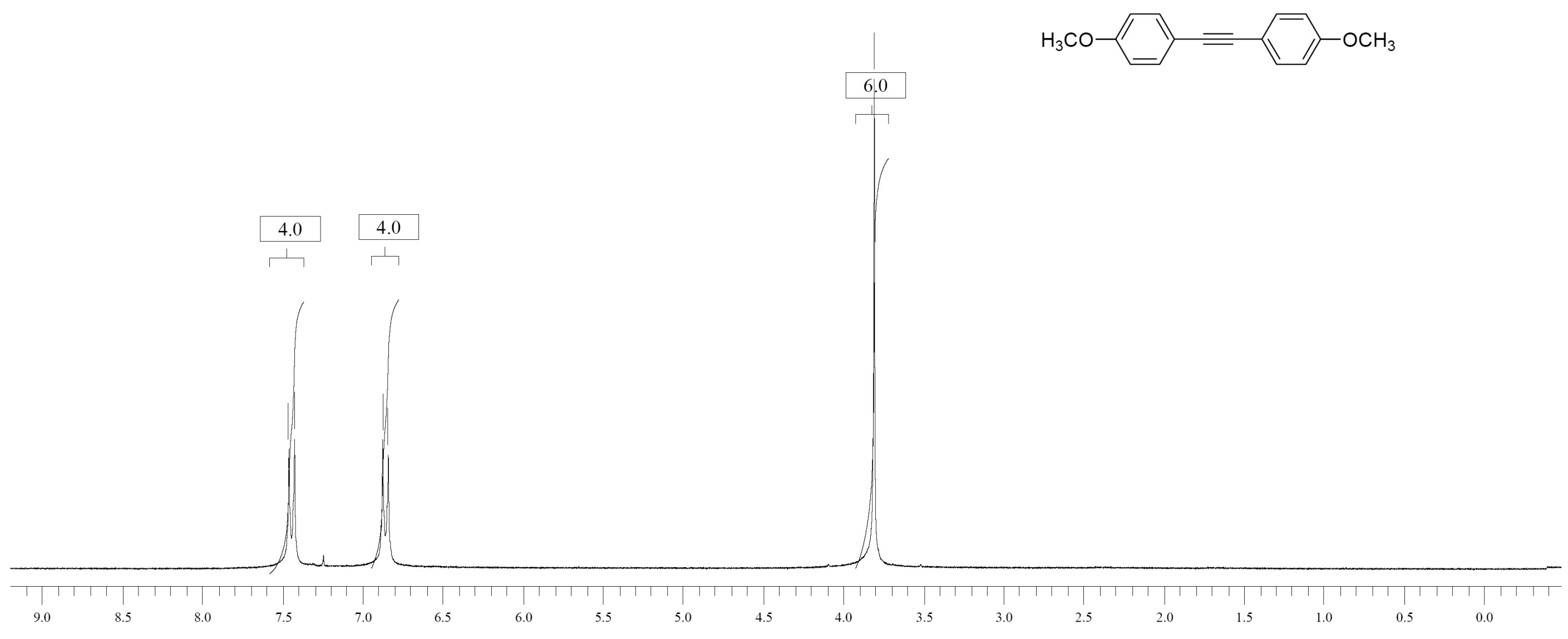



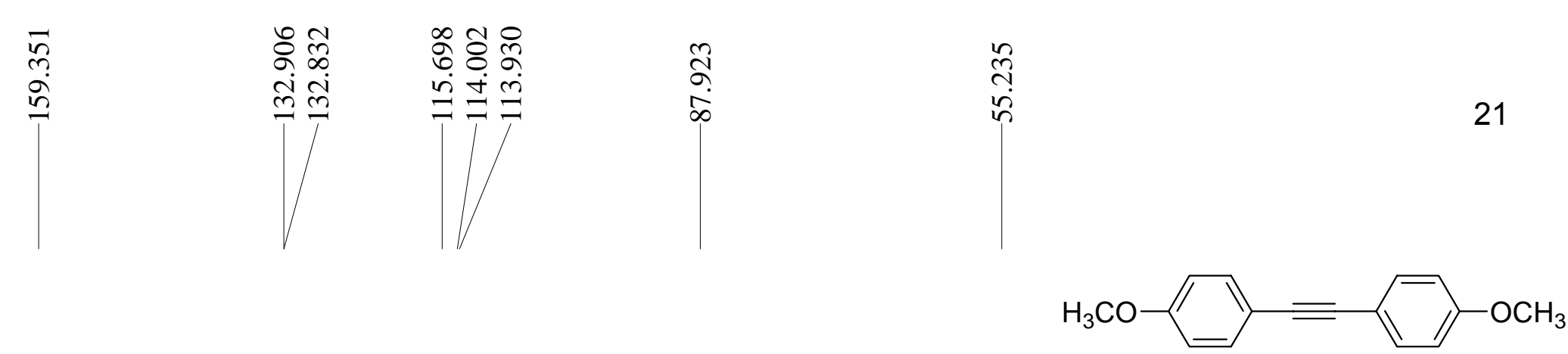

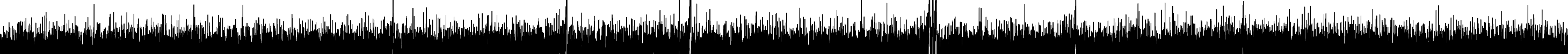
4. 


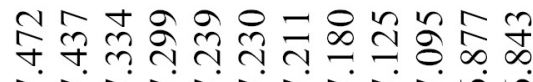
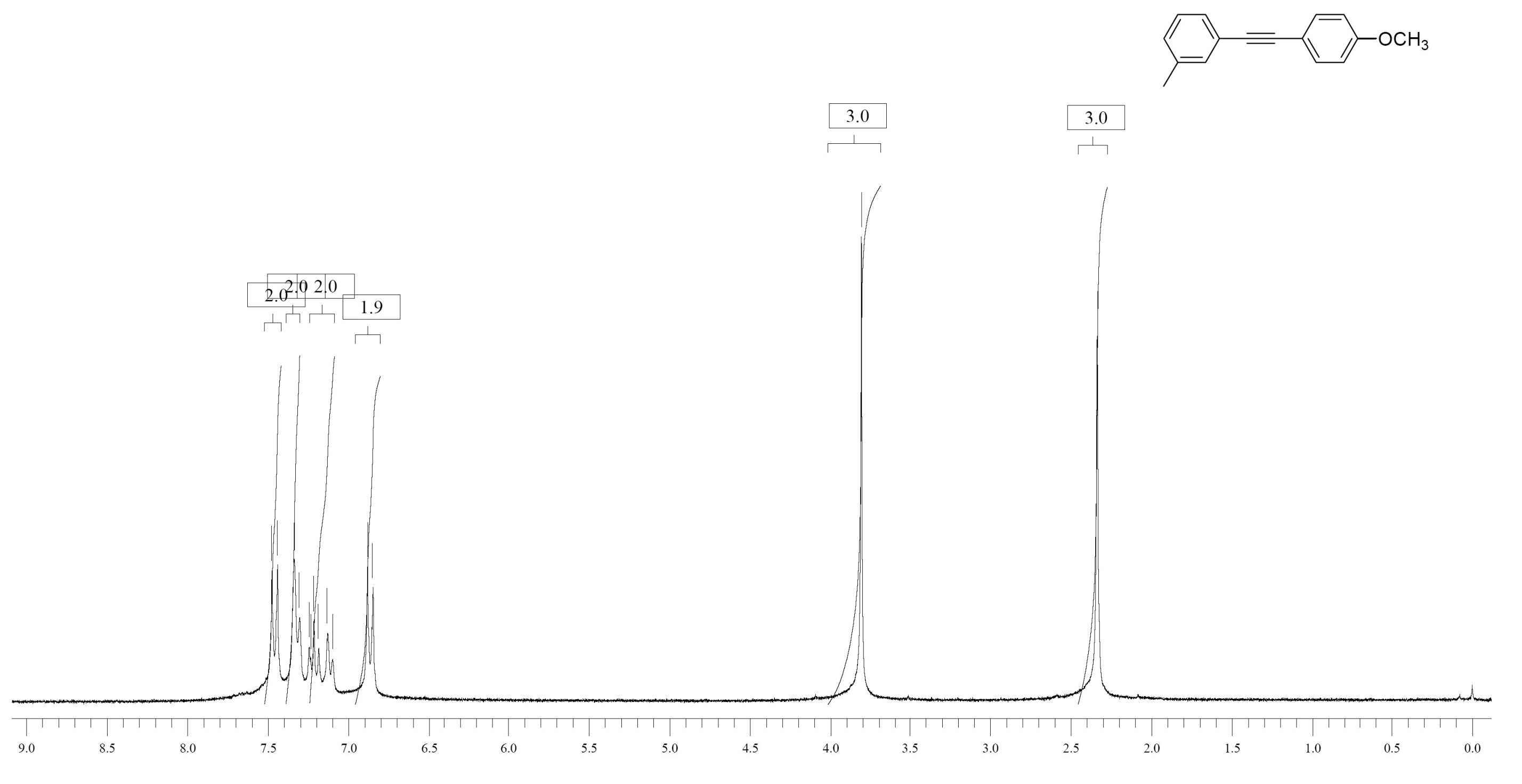

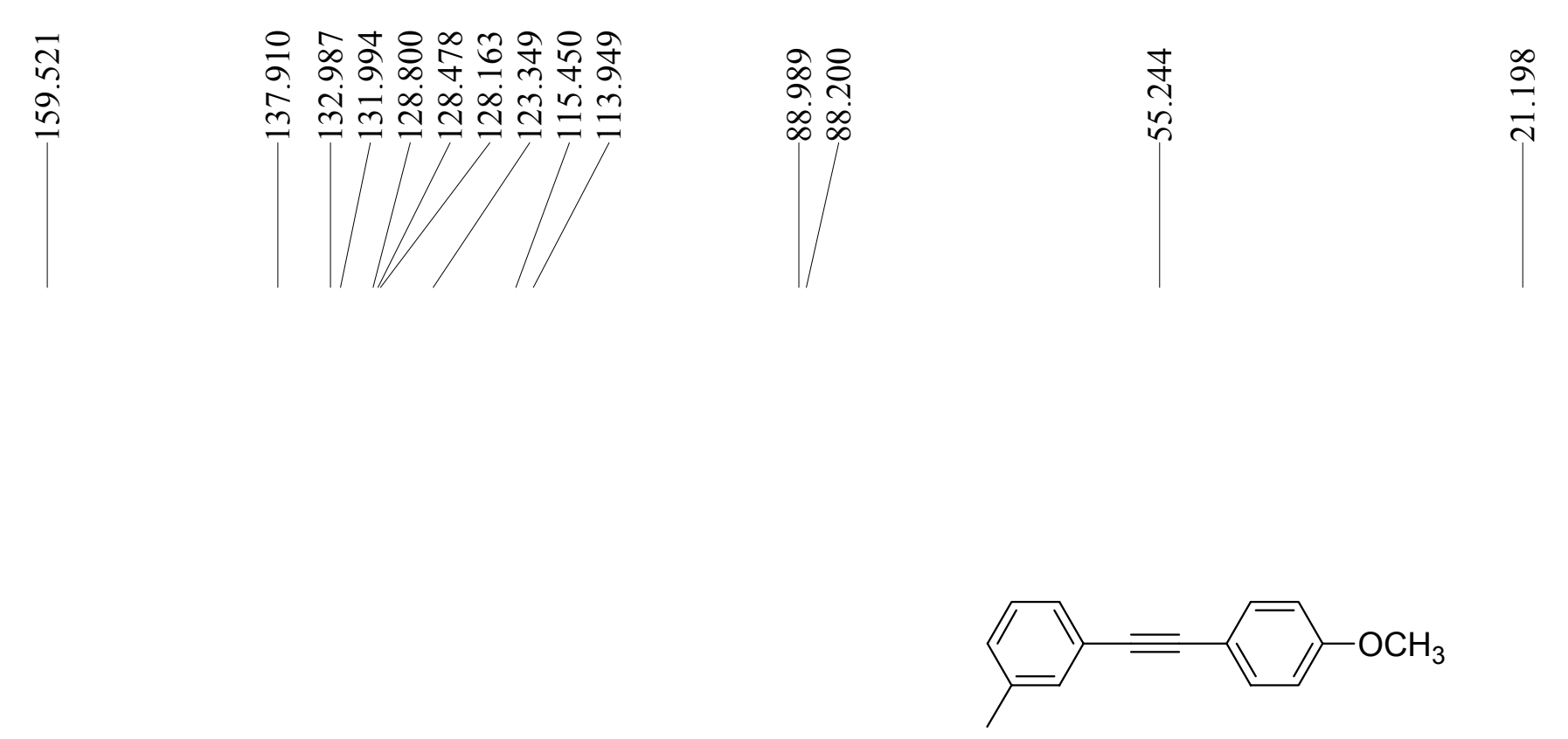


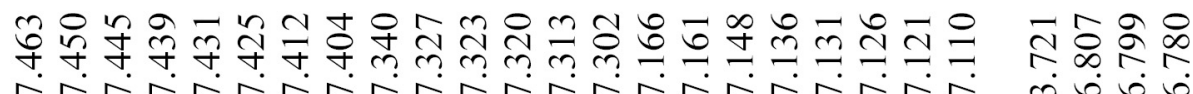
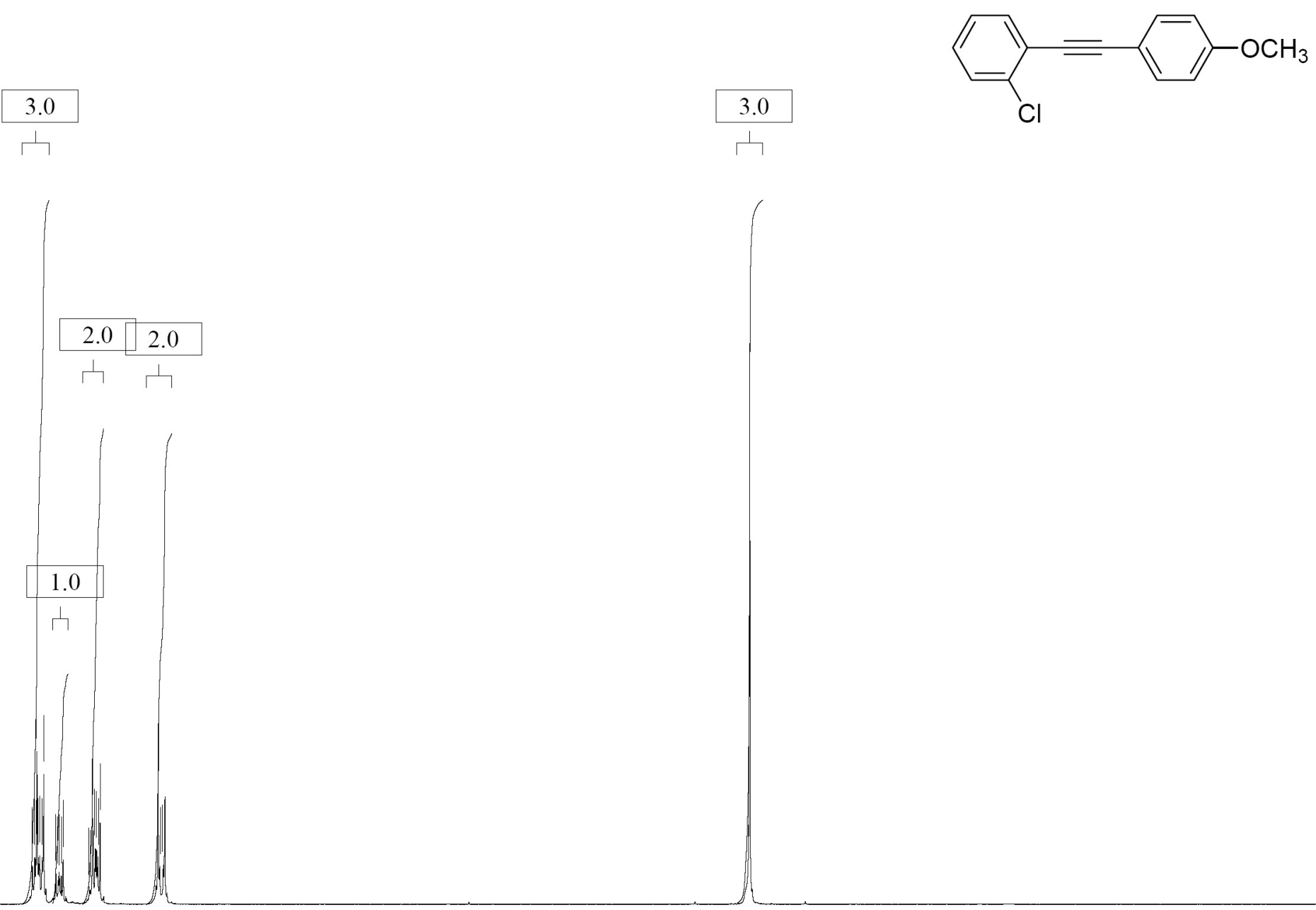

$\sqcap$ 


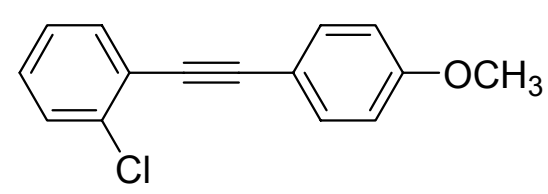




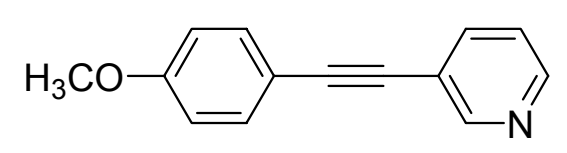



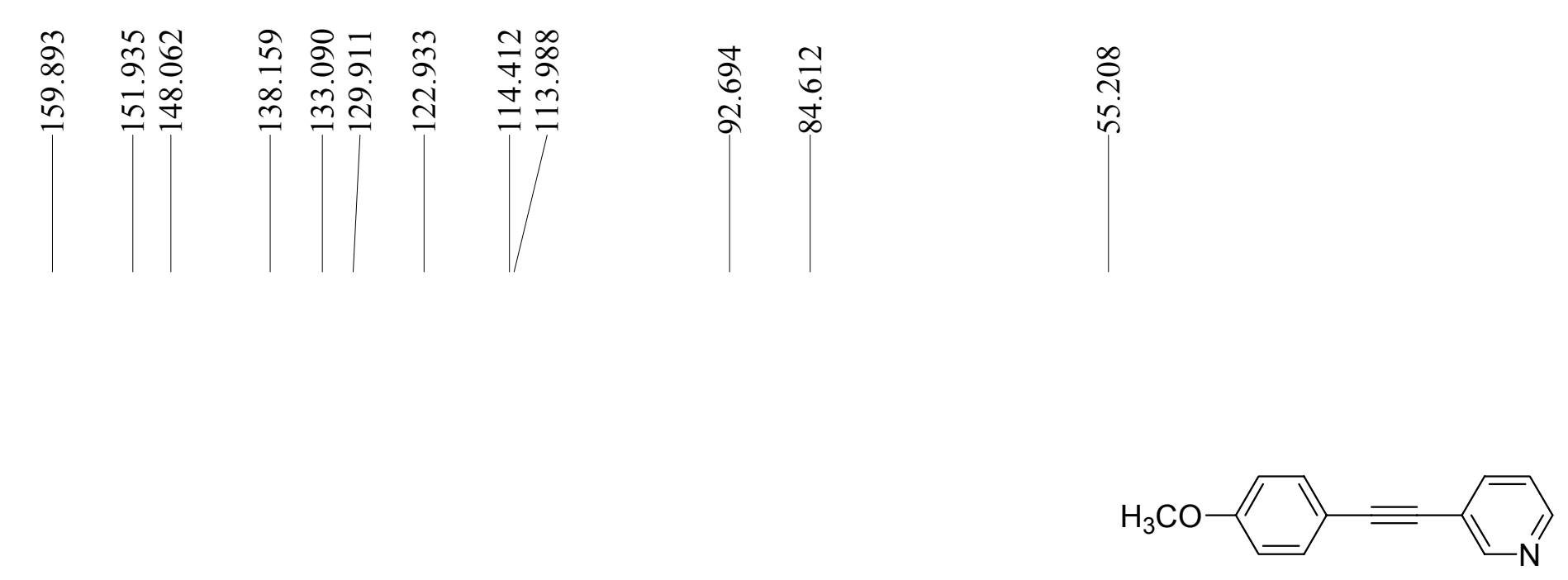

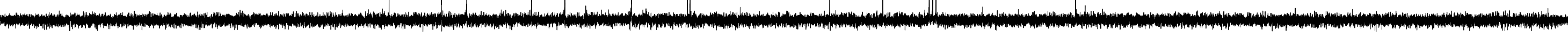




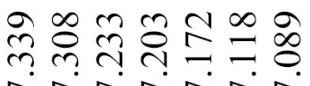

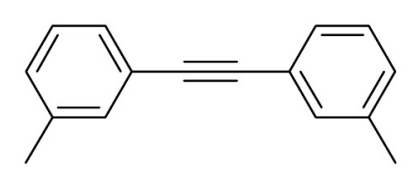




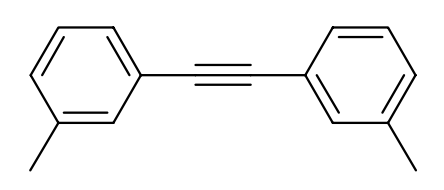




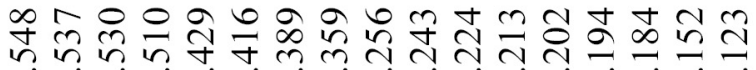

तर
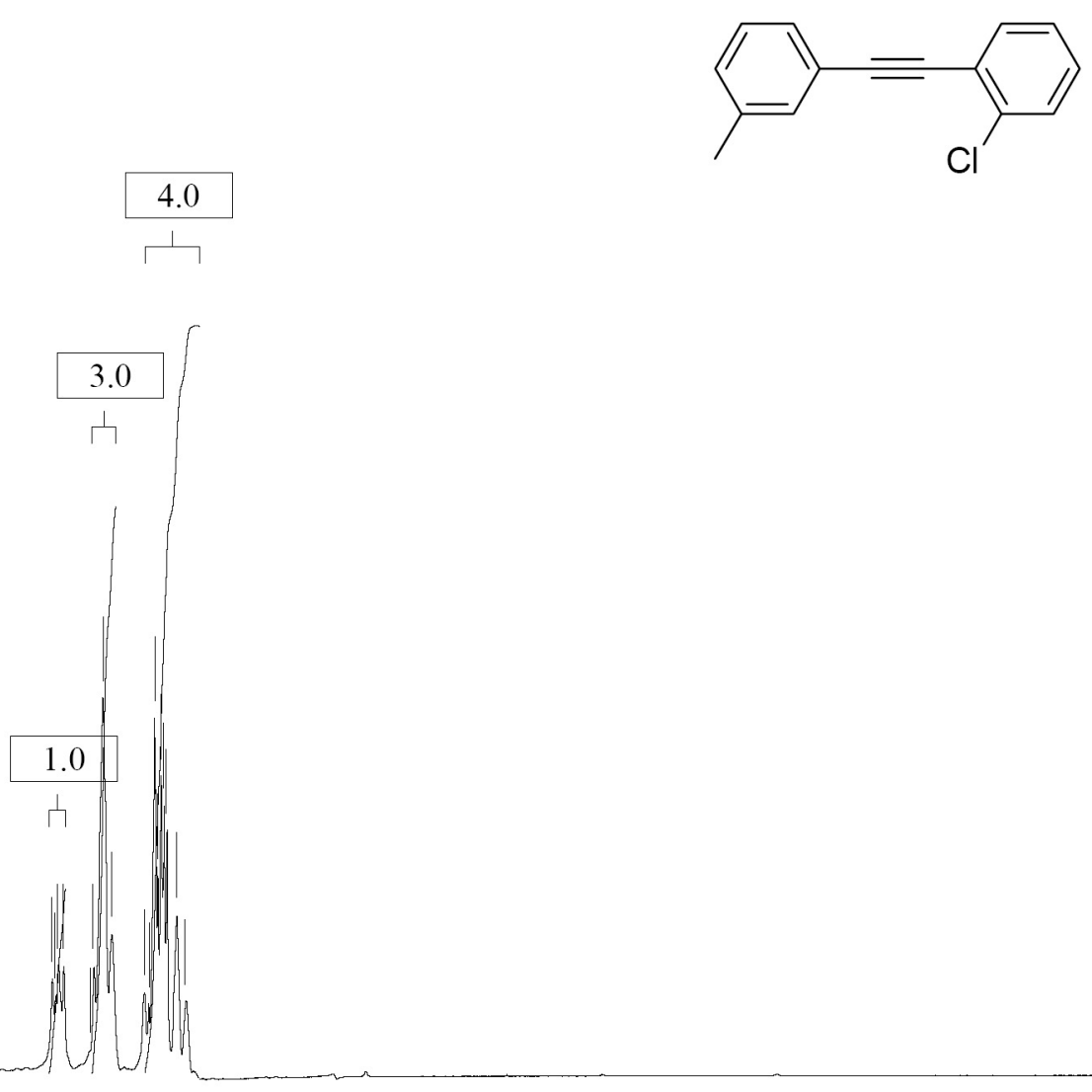


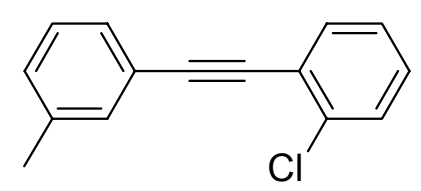




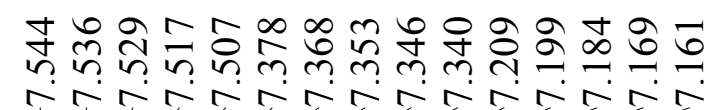
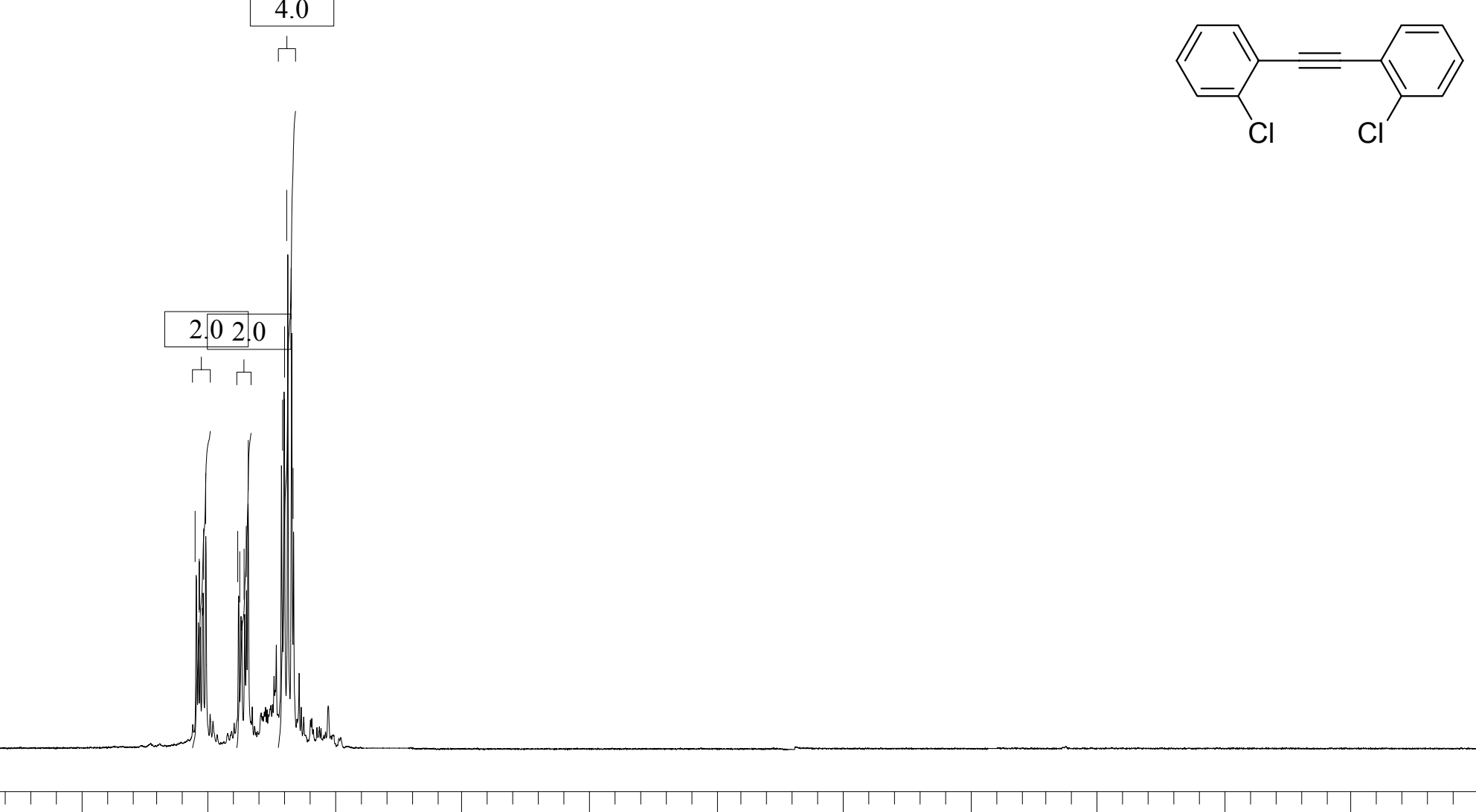


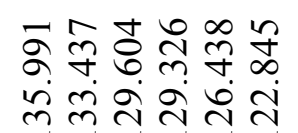

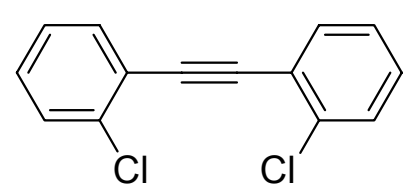




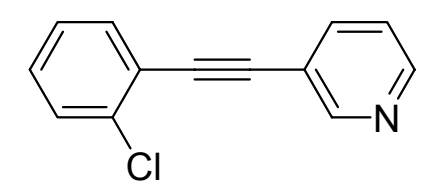




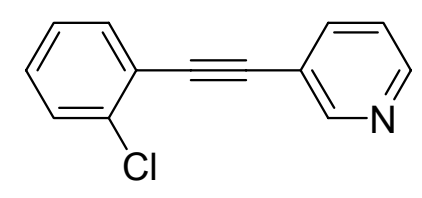




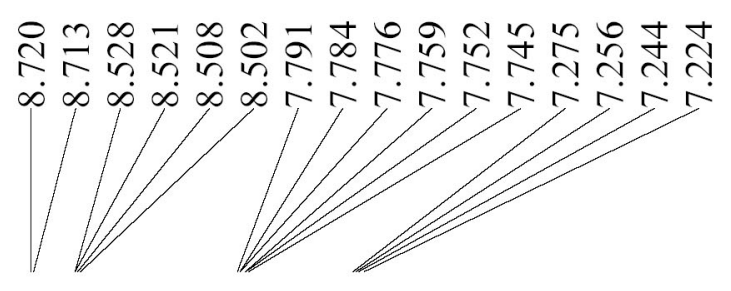

36

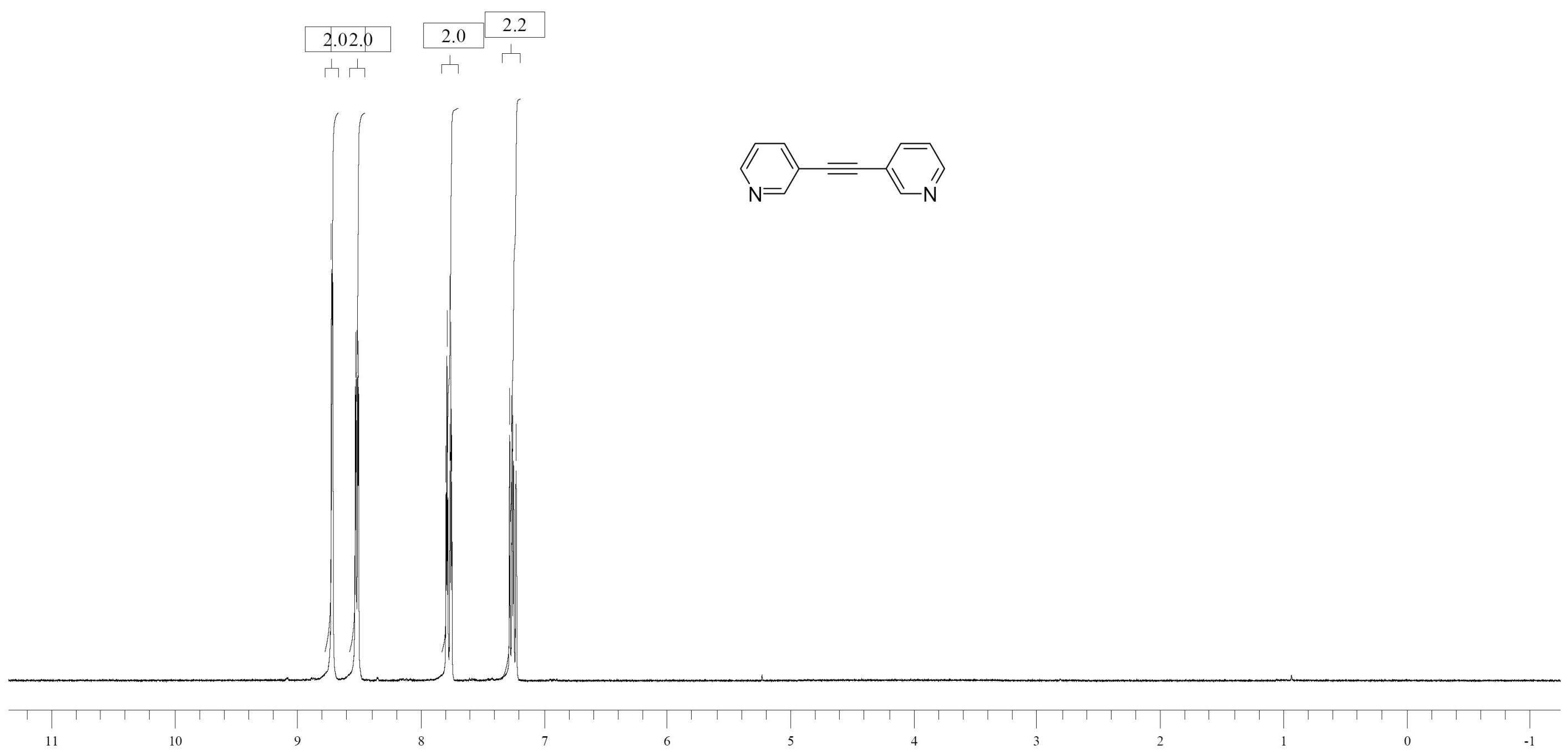


$\langle\overline{N=}=$ 